\title{
Curvature cones and the Ricci flow.
}

\author{
Thomas Richard
}

September 5, 2021

\begin{abstract}
This survey reviews some facts about nonnegativity conditions on the curvature tensor of a Riemannian manifold which are preserved by the action of the Ricci flow. The text focuses on two main points.

First we describe the known examples of preserved curvature conditions and how they have been used to derive geometric results, in particular sphere theorems.

We then describe some recent results which give restrictions on general preserved conditions.

The paper ends with some open questions on these matters.
\end{abstract}

The Ricci flow is the following evolution equation:

$$
\left\{\begin{array}{l}
\frac{\partial g}{\partial t}=-2 \operatorname{Ric}_{g(t)} \\
g(t)=g_{0}
\end{array}\right.
$$

where $(g(t))_{t \in[0, T)}$ is a one-parameter family of smooth Riemannian metrics on a fixed manifold $M$, and $g_{0}$ is a given smooth Riemannian metric on $M$. It was introduced by R. Hamilton in 1982 ([Ham82]), where it was used to study the topology of compact 3-manifolds with positive Ricci curvature. Analytically, the Ricci flow is a degenerate parabolic system. Existence and uniqueness for the Cauchy problem (1) have been established by Hamilton in the case where $M$ is compact and $g_{0}$ is smooth.

For a general introduction to the Ricci flow, see the books CK04, Top06, CLN06]. Since Hamilton's work, the Ricci flow has been used to solve various geometric problems. We refer to the previously cited books for examples. Here we will just briefly mention two of the biggest geometric achievements of Ricci flow:

- The proof of Thurston's Geometrization conjecture for 3-manifolds by G. Perelman, ([Per02, [Per03a, , Per03b] $)$, for an exposition of Perel-

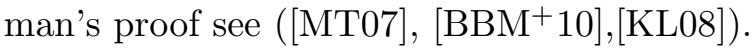

- The proof of the differentiable sphere theorem, by Brendle and Schoen ([BS09]), see also the books ([Bre10,, AH11] $)$. 
The objects we will be dealing with in this survey have little to do with Perelman's work, but were pivotal in the proof of the differentiable sphere theorem.

A priori estimates are among the most basic tools in the study of PDEs, one can divide them into two classes: integral estimates and pointwise estimates. Pointwise estimates often come from suitable maximum principles and are thus more often encountered in the realm of parabolic or elliptic equations. The Ricci flow being a geometric parabolic PDE, it is tempting to look for geometrically meaningful pointwise estimates for Ricci flows.

If one looks at Hamilton's foundational work, one sees that after having proven short time existence and derived some variation formulas for the Ricci flow, Hamilton proves the following result:

Proposition 0.1. Let $\left(M^{3}, g_{0}\right)$ be a compact 3-manifold with nonnegative Ricci curvature, then the solution $g(t)$ to (11) with initial condition $g_{0}$ satisfies $\operatorname{Ric}_{g(t)} \geq 0$ for all $t \geq 0$.

This is the kind of geometric pointwise estimate we will be concerned with. More precisely, we will try to gather what is known about various nonnegativity properties of the curvature of Riemannian manifold $\left(M, g_{0}\right)$ which remain valid for solutions $g(t)$ of the Ricci flow starting at $g_{0}$.

Let us now describe the contents of this paper. Section 1 sets the scene by introducing an abstract framework which allows us to consider nonnegativity conditions on the curvature as convex cones inside some vector space satisfying some invariance properties: the so-called curvature cones. We then describe how Hamilton's maximum principle characterizes which curvature cones lead to a nonnegativity condition on the curvature which is preserved under the action of the Ricci flow. In section 2 we review the various known examples of Ricci flow invariant curvature conditions, and see how they have been used to prove various sphere theorems. Section 3 deals with known restrictions on those Ricci flow invariant curvature conditions. In section 4 we gather some open questions on these matters.

Let us end this introduction with a disclaimer: this survey doesn't claim any originality in the treatment of the subject and draws heavily on the available litterature. Its main purpose is to gather results which were only available in separate articles before.

\section{Acknowledgements}

The author thanks Harish Seshadri for his advised comments on a preliminary version of this text. 


\section{Curvature cones}

\subsection{Definition and first properties}

Let us consider a Riemannian manifold $(M, g)$. We recall that its Riemann curvature tensor $R$ is the $(3,1)$ tensor defined by

$$
R(X, Y) Z=\left(\nabla_{Y} \nabla_{X}-\nabla_{X} \nabla_{Y}-\nabla_{[Y, X]}\right) Z
$$

where $\nabla$ is the Levi-Civitta connection canonically built from the metric $g$ and $X, Y, Z$ are vector fields on $M$. (Beware here that there is no universal convention for the sign of $R$.)

$R$ enjoys the following symmetries:

- $R(x, y) z=-R(y, x) z$,

- $R(x, y) z+R(y, z) x+R(z, x) y=0$,

- $g(R(x, y) z, t)=-g(R(x, y) t, z)$.

Using the symmetries of $R$, on can build at each $p \in M$ a symmetric endomorphism $\mathrm{R}$ of $\Lambda^{2} T_{p} M$ by

$$
g(\mathrm{R}(x \wedge y), z \wedge t)=g(R(x, y) z, t)
$$

for $x, y, z, t \in T_{p} M$. $\mathrm{R}$ is called the curvature operator of $(M, g)$.

Remark 1.1. Here, as in the rest of the paper, the inner product $g$ on $\Lambda^{2} T_{p} M$ is the one which comes from the metric $g$ by the following formula:

$$
g(x \wedge y, z \wedge t)=g(x, z) g(y, t)-g(x, t) g(y, z)
$$

(extended to non simple elements of $\Lambda^{2} T_{p} M$ by bilinearity).

We will use the same construction to endow $\Lambda^{2} \mathbb{R}^{n}$ with an inner product $\langle$,$\rangle coming from the standard inner product \langle$,$\rangle on \mathbb{R}^{n}$.

Definition 1.2. The space of algebraic curvature operators $S_{B}^{2} \Lambda^{2} \mathbb{R}^{n}$ is the space of symmetric endomorphisms $\mathrm{R}$ of $\Lambda^{2} \mathbb{R}^{n}$ which satisfy the first Bianchi identity:

$\forall x, y, z, t \in \mathbb{R}^{n} \quad\langle\mathrm{R}(x \wedge y), z \wedge t\rangle+\langle\mathrm{R}(z \wedge x), y \wedge t\rangle+\langle\mathrm{R}(y \wedge z), x \wedge t\rangle=0$.

Remark that $S_{B}^{2} \Lambda^{2} \mathbb{R}^{n}$ has a natural inner product given by:

$$
\langle\mathrm{R}, \mathrm{L}\rangle=\operatorname{trace}\left(\mathrm{RL}^{T}\right) .
$$

The space of algebraic curvature operators is the space of operators which satisfy the same symmetries as the curvature operators of Riemannian manifolds. As in the case of Riemannian manifolds, it is interesting to consider 
the Ricci morphism: $\rho: S_{B}^{2} \Lambda^{2} \mathbb{R}^{n} \rightarrow S^{2} \mathbb{R}^{n}$ which associates to an algebraic curvature operator $\mathrm{R}$ its Ricci tensor which is a symmetric operator on $\mathbb{R}^{n}$ defined by:

$$
\langle\rho(\mathrm{R}) x, y\rangle=\sum_{i=1}^{n}\left\langle\mathrm{R}\left(x \wedge e_{i}\right), y \wedge e_{i}\right\rangle
$$

where $\left(e_{i}\right)_{1 \leq i \leq n}$ is an orthonormal basis of $\mathbb{R}^{n}$. $\mathrm{R}$ is said to be Einstein if $\rho(\mathrm{R})$ is a multiple of the identity operator id $: \mathbb{R}^{n} \rightarrow \mathbb{R}^{n}$. Similarly, the scalar curvature of an algebraic curvature operator is just twice its trace.

The action of $O(n, \mathbb{R})$ on $\mathbb{R}^{n}$ induces the following action of $O(n, \mathbb{R})$ on $S_{B}^{2} \Lambda^{2} \mathbb{R}^{n}$ :

$$
\langle g . \mathrm{R}(x \wedge y), z \wedge t\rangle=\langle\mathrm{R}(g x \wedge g y), g z \wedge g t\rangle .
$$

The representation of $O(n, \mathbb{R})$ given by its action on $S_{B}^{2} \Lambda^{2} \mathbb{R}^{n}$ is decomposed into irreducible representations in the following way:

$$
S_{B}^{2} \Lambda^{2} \mathbb{R}^{n}=\mathbb{R} \mathrm{I} \oplus\left(S_{0}^{2} \mathbb{R}^{n} \wedge \mathrm{id}\right) \oplus \mathcal{W}
$$

where the space of Weyl curvature operators $\mathcal{W}$ is the kernel of the Ricci endomorphism $\rho: S_{B}^{2} \Lambda^{2} \mathbb{R}^{n} \rightarrow S^{2} \mathbb{R}^{n}$ and $S_{0}^{2} \mathbb{R}^{n} \wedge$ id is the image of the space of traceless endomorphims of $\mathbb{R}^{n}$ under the application $A_{0} \mapsto A_{0} \wedge$ id. The wedge product of two symmetric operators $A, B: \mathbb{R}^{n} \rightarrow \mathbb{R}^{n}$ is defined by

$$
(A \wedge B)(x \wedge y)=\frac{1}{2}(A x \wedge B y+B x \wedge A y) .
$$

This corresponds to half the Kulkarni-Nomizu product of $A$ and $B$ viewed as quadratic forms. In dimension 2, only the first summand of (3) exists. In dimension 3 the $\mathcal{W}$ factor is 0 . Starting in dimension 4 , all three components exist.

When needed, we will write $\mathrm{R}=\mathrm{R}_{\mathrm{I}}+\mathrm{R}_{0}+\mathrm{R}_{\mathcal{W}}$ the decomposition of a curvature operator along the three irreducible components of (3).

Definition 1.3. A curvature cone is a closed convex cone $\mathcal{C} \subset S_{B}^{2} \Lambda^{2} \mathbb{R}^{n}$ which is invariant under the action of $O(n, \mathbb{R})$ given by (2).

Definition 1.4. A curvature cone is said to be nonnegative if it contains the identity operator $\mathrm{I}: \Lambda^{2} \mathbb{R}^{n} \rightarrow \Lambda^{2} \mathbb{R}^{n}$ in its interior.

This definition can be tracked back to the article Gro91] of M. Gromov. One should notice that we require the cone to be invariant under the full orthogonal group $O(n, \mathbb{R})$, rather than under the special orthogonal group $S O(n, \mathbb{R})$. This makes a difference only in dimension 4 , where the action of $S O(4, \mathbb{R})$ on the space of Weyl tensors is not irreducible. The behavior of these "oriented" curvature cones will be briefly addressed in section 3.2 .

Each nonnegative curvature cone $\mathcal{C}$ can be used to define a nonnegativity condition on the curvature of Riemannian manifolds. The curvature operator $\mathrm{R}$ of a Riemannian manifold $(M, g)$ is a section of the bundle $S_{B}^{2} \Lambda^{2} T M$ 
which is built from $T M$ the same way $S_{B}^{2} \Lambda^{2} \mathbb{R}^{n}$ is built from $\mathbb{R}^{n}$. For each $x \in M$, one can choose an orthonormal basis of $T_{x} M$ to build an isomorphism between $S_{B}^{2} \Lambda^{2} T_{x} M$ and $S_{B}^{2} \Lambda^{2} \mathbb{R}^{n}$. Thanks to the $O(n, \mathbb{R})$-invariance of $\mathcal{C}$, this allows us to embed $\mathcal{C}$ in $S_{B}^{2} \Lambda^{2} T_{x} M$ in a way which is independent of the basis of $T_{x} M$ we started with.

Definition 1.5. Let $\mathcal{C}$ be a nonnegative curvature cone.

A Riemannian manifold $(M, g)$ has $\mathcal{C}$-nonnegative curvature if for any $x \in M$ the curvature operator of $(M, g)$ at $x$ belongs to the previously discussed embedding of $\mathcal{C}$ in $S_{B}^{2} \Lambda^{2} T_{x} M$.

Similarly, $(M, g)$ has positive $\mathcal{C}$-curvature if its curvature operator at each point is in the interior of $\mathcal{C}$.

Let us give a couple of examples of curvature cones:

- $\mathbb{R} I, S_{0}^{2} \mathbb{R}^{n} \wedge$ id and $\mathcal{W}$ are curvature cones, which are not nonnegative.

- $\{\mathrm{R} \mid$ trace $\mathrm{R} \geq 0\}$ is a nonnegative curvature curvature cone. The corresponding nonnegativity condition is "nonnegative scalar curvature".

- Similarly the conditions "nonnegative Ricci curvature", "nonnegative sectionnal curvature" and "nonnegative curvature operator" define nonnegative curvature cones.

With these examples in mind we can go back to the definition and try to explain its significance.

- The fact that $\mathcal{C}$ is $O(n, \mathbb{R})$-invariant is mandatory to be able make sense of the " $\mathcal{C}$ nonnegative curvature" condition.

- Requiring that $\mathcal{C}$ is a cone ensures that the associated geometric condition is invariant under scalings, which is expected from a nonnegativity condition on the curvature.

- Asking for I to be in the interior of $\mathcal{C}$ is equivalent to requiring that the round sphere has positive $\mathcal{C}$-curvature. It also ensure that the "positive $\mathcal{C}$-curvature" condition is stable with respect to $C^{2}$ perturbations on the space of Riemannian metrics.

- It is not so clear why one should ask for convexity of $\mathcal{C}$, however it is satisfied by all classical curvature conditions and turns out to be a crucial hypothesis when dealing with Hamilton's maximum principle (see next section).

We have the following elementary observation, whose elementary proof is not written anywhere as far as the author knows:

Proposition 1.6. If $\mathcal{C}$ is a nonnegative curvature cone which is not the full space $S_{B}^{2} \Lambda^{2} \mathbb{R}^{n}$, then $\mathcal{C} \subset\{\mathrm{R} \mid$ trace $\mathrm{R} \geq 0\}$. 
Proof. Let us assume that $\mathcal{C}$ contains a curvature operator $\mathrm{R}$ with negative trace. Consider the average $\tilde{\mathrm{R}}=\int_{O(n)} g \cdot \mathrm{R} d g$ of the $O(n)$-orbit of $\mathrm{R}$ with respect to the Haar measure $d g$ on $O(n)$.

The irreducibility of $\mathcal{W}$ and $S_{0}^{2} \mathbb{R}^{n} \wedge$ id imply that the projection of $\tilde{\mathrm{R}}$ on these subspaces vanishes, thus $\tilde{\mathrm{R}}=\lambda$ I where $\lambda<0$ since trace $\tilde{\mathrm{R}}=$ trace $\mathrm{R}<$ 0 . Hence $\mathcal{C}$ contains the whole line $\mathbb{R} I$ and since $\mathrm{I}$ is in the interior of $\mathcal{C}$, this implies that $\mathcal{C}=S_{B}^{2} \Lambda^{2} \mathbb{R}^{n}$.

\subsection{Cones which behave well under the Ricci flow}

We now consider the interplay between these curvature cones and the Ricci flow. If $(M, g(t))$ is a Ricci flow, Hamilton has proved in Ham86 that the curvature operator $\mathrm{R}_{g(t)}$ of $(M, g(t))$ satisfies the following evolution equation:

$$
\frac{\partial \mathrm{R}_{g(t)}}{\partial t}=\Delta_{g(t)} \mathrm{R}_{g(t)}+2 Q\left(\mathrm{R}_{g(t)}\right)
$$

where $Q$ is the $O(n, \mathbb{R})$ quadratic vector field on $S_{B}^{2} \Lambda^{2} \mathbb{R}^{n}$ defined by:

$$
Q(\mathrm{R})=\mathrm{R}^{2}+\mathrm{R}^{\#}
$$

Here, $\mathrm{R}^{2}$ is just the square of $\mathrm{R}$ seen as an endomorphism of $\Lambda^{2} \mathbb{R}^{n} \cdot \mathrm{R}^{\#}$ is defined in the following way:

$$
\left\langle\mathrm{R}^{\#} \eta, \eta\right\rangle=-\frac{1}{2} \operatorname{trace}\left(\operatorname{ad}_{\omega} \circ \mathrm{R} \circ \operatorname{ad}_{\omega} \circ \mathrm{R}\right)
$$

where $\operatorname{ad}_{\omega}: \Lambda^{2} \mathbb{R}^{n} \rightarrow \Lambda^{2} \mathbb{R}^{n}$ is the endomorphism $\eta \mapsto[\omega, \eta]$. In the previous formula, the Lie bracket [, ] on $\Lambda^{2} \mathbb{R}^{n}$ comes from its identification with $\mathfrak{s o}(n, \mathbb{R})$ given by:

$$
x \wedge y \mapsto(u \mapsto\langle x, u\rangle y-\langle y, u\rangle x) .
$$

This expression for $\mathrm{R}^{\#}$ can be found in BW08.

We will sometimes use the bilinear map $B$ associated to the quadratic map $Q$, it is defined in the usual way:

$$
B\left(\mathrm{R}_{1}, \mathrm{R}_{2}\right)=\frac{1}{2}\left(Q\left(\mathrm{R}_{1}+\mathrm{R}_{2}\right)-Q\left(\mathrm{R}_{1}\right)-Q\left(\mathrm{R}_{2}\right)\right) .
$$

We are now ready to define a Ricci flow invariant curvature cone:

Definition 1.7. A curvature cone $\mathcal{C}$ is said to be Ricci flow invariant if for any $\mathrm{R}$ in the boundary $\partial \mathcal{C}$ of $\mathcal{C}, Q(\mathrm{R}) \in T_{\mathrm{R}} \mathcal{C}$, the tangent cone at $\mathrm{R}$ to $\mathcal{C}$.

Remark 1.8. In other words, a cone is Ricci flow invariant if at every $\mathrm{R} \in \partial \mathcal{C}$, $Q(\mathrm{R})$ points towards the inside of $\mathcal{C}$.

This condition is equivalent to the fact that the solutions to the ODE $\frac{d}{d t} \mathrm{R}=Q(\mathrm{R})$ which start inside $\mathcal{C}$ stay in $\mathcal{C}$ for positive times. 
Hamilton's maximum principle (see [Ham86]) implies:

Theorem 1.9. Let $\mathcal{C}$ be a Ricci flow invariant curvature cone. If $(M, g(t))_{t \in[0, T)}$ is a Ricci flow on a compact manifold such that $(M, g(0))$ has $\mathcal{C}$-nonnegative curvature, then for $t \in[0, T),(M, g(t))$ has $\mathcal{C}$-nonnegative curvature.

Remark 1.10. It could happen that a nonnegativity condition is preserved under the Ricci flow while the associated cone is not Ricci flow invariant according to our definition, however such examples are not known to exist, as far as the knowledge of the author goes.

\section{Examples of Ricci flow invariant curvature cones}

\subsection{First examples}

We will first give two prototypes of Ricci flow invariant cones. The discovery of the Ricci flow invariance of these cones is due to Hamilton.

Proposition 2.1. $\mathcal{C}_{\text {Scal }}=\{\mathrm{R} \mid$ trace $\mathrm{R} \geq 0\}$ is a Ricci flow invariant nonnegative curvature cone.

Proof. The boundary of $\mathcal{C}_{\text {Scal }}$ is the hyperplane $\partial \mathcal{C}_{\text {Scal }}=\{\mathrm{R} \mid$ trace $\mathrm{R}=0\}$. The tangent cone at any $\mathrm{R} \in \partial \mathcal{C}_{\text {Scal }}$ is actually (since $\mathcal{C}_{\text {Scal }}$ is a half space) $\mathcal{C}_{\text {Scal }}$ itself.

Thus we only need to show that for any $\mathrm{R}$ whose trace vanishes, trace $Q(\mathrm{R}) \geq$ 0 . This is easily seen to be true thanks to the following formula (which is actually valid for any $R$ ):

$$
\operatorname{trace} Q(\mathrm{R})=2|\rho(\mathrm{R})|^{2} \geq 0 .
$$

(Recall that $\rho: S_{B}^{2} \Lambda^{2} \mathbb{R}^{n} \rightarrow S^{2} \mathbb{R}^{n}$ is the map which sends a curvature operator to its Ricci endomorphism.)

Proposition 2.2. $\mathcal{C}_{P C O}=\left\{\mathrm{R} \mid \forall \omega \in \Lambda^{2} \mathbb{R}^{2},\langle\mathrm{R} \omega, \omega\rangle \geq 0\right\}$ is a Ricci flow invariant nonnegative curvature cone.

Proof. A nonnegative curvature operator $\mathrm{R}$ belongs to the boundary of $\mathcal{C}_{P C O}$ if and only if $\operatorname{Ker}(\mathrm{R}) \neq\{0\}$. In this case:

$$
T_{\mathrm{R}} \mathcal{C}_{P C O}=\{\mathrm{L} \mid \forall \omega \in \operatorname{Ker}(\mathrm{R}),\langle\mathrm{L} \omega, \omega\rangle \geq 0\} .
$$

Thus we only need to show that for any $\omega \in \operatorname{Ker}(\mathrm{R}),\langle Q(\mathrm{R}) \omega, \omega\rangle \geq 0$. Recall that $Q(\mathrm{R})=\mathrm{R}^{2}+\mathrm{R}^{\#}$. By the symmetry of $\mathrm{R},\left\langle\mathrm{R}^{2} \omega, \omega\right\rangle=0$, thus we only need to deal with $\left\langle\mathrm{R}^{\#} \omega, \omega\right\rangle$. In order to do this we choose an 
orthonormal basis $\eta_{i}$ of $\Lambda^{2} \mathbb{R}^{n}$ consisting of eigenvectors $\mathrm{R}$ with associated eigenvalues $\lambda_{i} \geq 0$. We compute:

$$
\begin{aligned}
\left\langle\mathrm{R}^{\#} \omega, \omega\right\rangle & =-\frac{1}{2} \operatorname{trace}\left(\operatorname{ad}_{\omega} \circ \mathrm{R} \circ \operatorname{ad}_{\omega} \circ \mathrm{R}\right) \\
& =-\frac{1}{2} \sum_{i}\left\langle\left[\omega, \mathrm{R}\left[\omega, \mathrm{R} \eta_{i}\right]\right], \eta_{i}\right\rangle \\
& =\frac{1}{2} \sum_{i}\left\langle\mathrm{R}\left[\omega, \mathrm{R} \eta_{i}\right],\left[\omega, \eta_{i}\right]\right\rangle \\
& =\frac{1}{2} \sum_{i} \lambda_{i}\left\langle\mathrm{R}\left[\omega, \eta_{i}\right],\left[\omega, \eta_{i}\right]\right\rangle \\
& \geq 0
\end{aligned}
$$

since $\mathrm{R}$ is nonnegative.

Let us now briefly discuss the status of the most important curvature cones in Riemannian geometry: the cone of operators with nonnegative sectional curvature and the cone of curvature operators with nonnegative Ricci curvature. They are Ricci flow invariant in dimension 3. However starting with dimension 4 these cone are not Ricci flow invariant.

For the cone $\mathcal{C}_{\text {Ric }}$ of curvature operators with nonnegative Ricci curvature, an even stronger result is actually available: there exists compact Kaehler surfaces $\left(M^{4}, g_{0}\right)$ with nonnegative Ricci curvature whose Ricci flow $(g(t))_{t>0}$ has negative Ricci curvature in some directions. This has been proven by Maximo in Max11.

For the cone $\mathcal{C}_{\text {sec }}$ of curvature operators with nonnegative sectional curvature, it is quite easy to find an explicit point in the boundary of $\mathcal{C}_{\mathrm{sec}}$ where $Q$ doesn't point inside $\mathcal{C}_{\text {sec }}$.

Let $\mathrm{R}$ be the curvature operator of $\mathbb{C P}^{n}(n \geq 2)$ normalized to have sectional curvature between 1 and 4 . Then $\tilde{R}=\mathrm{R}-\mathrm{I} \in \partial \mathcal{C}_{\text {sec }}$. Let $\Pi$ be a plane in $\mathbb{C}^{n}$ whose sectional curvature is 1 . Let $\left(e_{1}, e_{2}\right)$ be a (real) basis of $\Pi$ (note that $\mathbb{C} e_{1}$ and $\mathbb{C}_{2}$ must be orthogonal in order to ensure that the sectional curvature of $\Pi$ for $R$ is 1$)$. The sectional of $\Pi$ for $\tilde{R}$ is 0 . Thus, if $Q(\tilde{\mathrm{R}})$ was to point inside $\mathcal{C}_{\text {sec }}$, the sectional curvature of $\Pi$ for $Q(\tilde{\mathrm{R}})$ would be nonnegative. But a quick computation shows that $Q(\tilde{\mathrm{R}})=$ $2(n+1) \mathrm{R}-(2 n+5) \mathrm{I}$. Hence the sectional curvature of $\Pi$ with respect to $Q(\tilde{\mathrm{R}})$ is actually -3 . This shows that $\mathcal{C}_{\text {sec }}$ is not Ricci flow invariant starting in dimension 4 .

Thus starting in dimension 4, we need to consider more exotic curvature cones. We will here define the most important of these Ricci flow invariant curvature cones.

We start with the cone of 2-nonnegative curvature operators: 
Definition 2.3. $\mathcal{C}_{2 P C O}$ is the cone of 2-nonnegative curvature operators, more precisely it consists of all curvature operators whose two lowest eigenvalues have positive sum.

The other cones are derived from the "positive isotropic curvature" (PIC) condition, introduced by Micallef and Moore in [MM88] as an obstruction to the existence of area minimizing two spheres in compact Riemannian manifolds.

We extend any curvature operator $\mathrm{R}$ to $\Lambda^{2} \mathbb{C}^{n}$ in a complex linear way. We also extend the inner products on $\mathbb{R}^{n}$ and $\Lambda^{2} \mathbb{R}^{n}$ in a complex bilinear way to $\mathbb{C}^{n}$ and $\Lambda^{2} \mathbb{C}^{n}$. The resulting complex symmetric bilinear forms will still be denoted by $\langle$,$\rangle . Note that these symmetric complex bilinear forms$ admit isotropic vectors. A subspace $V \subset \mathbb{C}^{n}$ is said to be totally isotropic if every very vector $v \in V$ is isotropic (satisfies $\langle v, v\rangle=0$ ).

The complex sectional curvature of a complex plane $\Pi$ in $\mathbb{C}^{n}$ is defined by:

$$
K^{\mathbb{C}}(\Pi)=\langle\mathrm{R}(u \wedge v), \bar{u} \wedge \bar{v}\rangle
$$

where $(u, v)$ is a basis of $\Pi$ which is orthonormal basis with respect to the hermitian inner product on $\mathbb{C}^{n}$ induced by $\langle$,$\rangle and complex conjugation.$

Definition 2.4. A curvature operator $\mathrm{R}$ is said to have nonnegative isotropic curvature (in short: is $\overline{P I C}$ ) if $K^{\mathbb{C}}(\Pi) \geq 0$ for every totally isotropic complex plane $\Pi$. The cone of $\overline{P I C}$ operators is denoted by $\mathcal{C}_{I C}$.

Remark 2.5. The following characterization of PIC curvature operators is useful: $\mathrm{R}$ is $\overline{P I C}$ if and only if for every orthonormal 4 -frame $\left(e_{1}, e_{2}, e_{3}, e_{4}\right)$ :

$$
\mathrm{R}_{1313}+\mathrm{R}_{1414}+\mathrm{R}_{2323}+\mathrm{R}_{2424}-2 \mathrm{R}_{1234} \geq 0
$$

where $R_{i j k l}$ stands for $\left\langle\mathrm{R}\left(e_{i} \wedge e_{j}\right), e_{k} \wedge e_{l}\right\rangle$.

Definition 2.6. A curvature operator $\mathrm{R}: \Lambda^{2} \mathbb{R}^{n} \rightarrow \Lambda^{2} \mathbb{R}^{n}$ is $\overline{P I C 1}$ if its natural extension $\tilde{\mathrm{R}}: \Lambda^{2} \mathbb{R}^{n+1} \rightarrow \Lambda^{2} \mathbb{R}^{n+1}$ is $\overline{\text { PIC }}$. The cone of $\overline{\text { PIC1 }}$ operators is denoted by $\mathcal{C}_{I C 1}$.

Definition 2.7. A curvature operator $\mathrm{R}: \Lambda^{2} \mathbb{R}^{n} \rightarrow \Lambda^{2} \mathbb{R}^{n}$ is $\overline{\text { PIC2 }}$ if its natural extension $\tilde{\mathrm{R}}: \Lambda^{2} \mathbb{R}^{n+2} \rightarrow \Lambda^{2} \mathbb{R}^{n+2}$ is $\overline{P I C}$. The cone of $\overline{P I C 2}$ operators is denoted by $\mathcal{C}_{I C 2}$.

One obviously has that $\mathcal{C}_{I C 2} \subset \mathcal{C}_{I C 1} \subset \mathcal{C}_{I C}$.

Theorem 2.8. The nonnegative curvature cones $\mathcal{C}_{2 P C O}, \mathcal{C}_{I C}, \mathcal{C}_{I C 1}$ and $\mathcal{C}_{I C 2}$ are Ricci flow invariant.

The invariance of $\mathcal{C}_{2 P C O}$ is due to Chen ([Che91]). The invariance of $\mathcal{C}_{I C}$ is due to Brendle and Schoen ([BS09]) and, independently, Nguyen (Ngu10]). The invariance of $\mathcal{C}_{I C 1}$ and $\mathcal{C}_{I C 2}$ follows immediately from the 


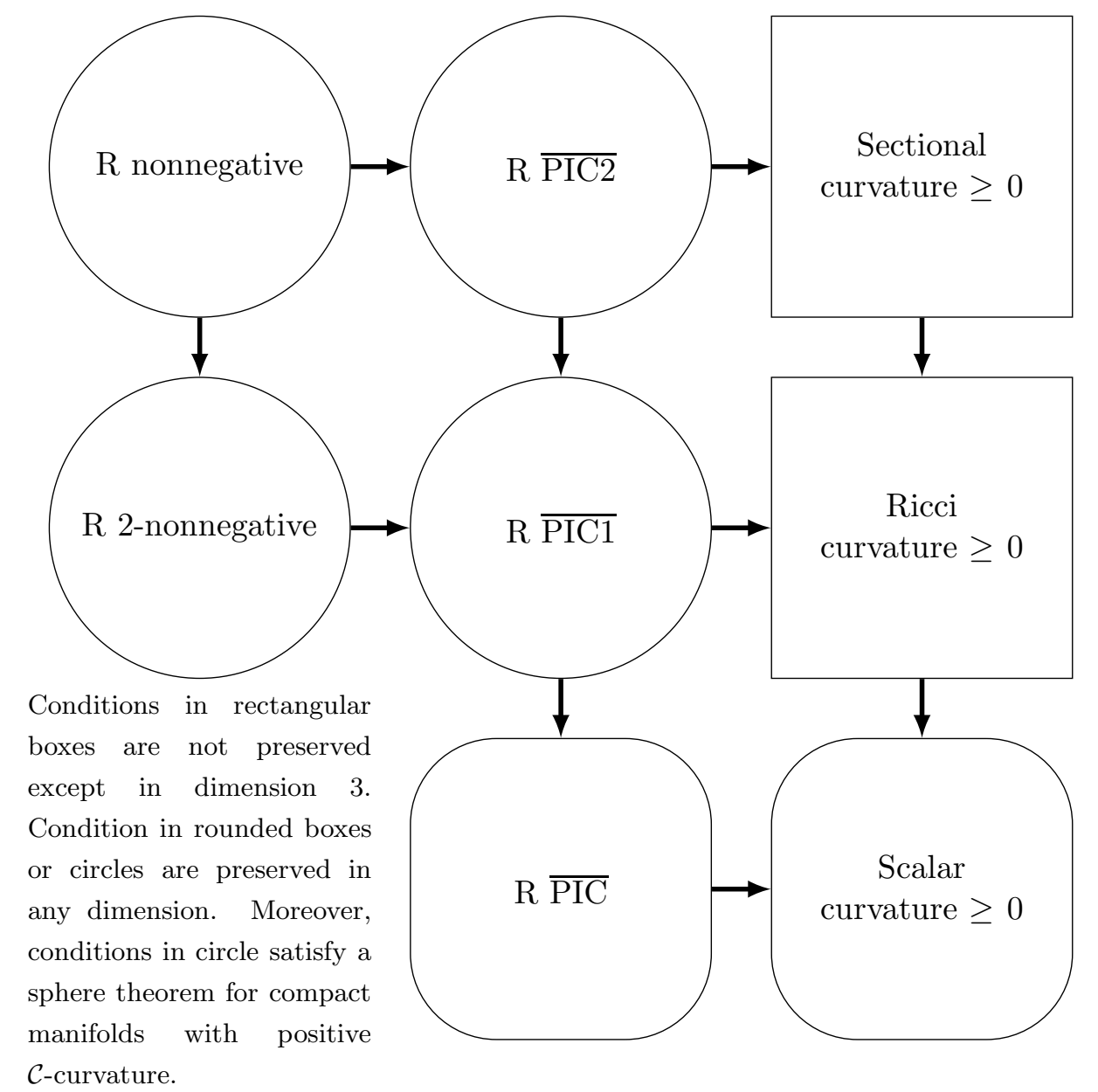

Figure 1: Behavior of classical curvature conditions under Ricci flow.

invariance of $\mathcal{C}_{I C}$ and the fact that the $\mathrm{ODE} \mathrm{R}^{\prime}=Q(\mathrm{R})$ respects product structures. It was first pointed out in [BS09].

We will see in the next section a unified proof of the invariance of these four cones, due to Wilking.

\subsection{Wilking's construction}

We now describe a construction due to Wilking which recovers most of the Ricci flow invariant curvature conditions in one relatively easy proof. This construction was published in Wil13.

Before stating the criterion, recall that $\Lambda^{2} \mathbb{R}^{n}$ is naturally isomorphic to $\mathfrak{s o}(n, \mathbb{R})$. And not the action of $S O(n, \mathbb{R})$ on $\Lambda^{2} \mathbb{R}^{n}$ is actually just the adjoint action of $S O(n, \mathbb{R})$ on its Lie algebra $\mathfrak{s o}(n, \mathbb{R})$, thus we will denote the action an element $g \in S O(n, \mathbb{R})$ on $\omega \in \mathfrak{s o}(n, \mathbb{R}) \simeq \Lambda^{2} \mathbb{R}^{n}$ by $\operatorname{Ad}_{g} \omega$, moreover we 
will denote the Lie bracket $[\omega, \eta]=\operatorname{ad}_{\omega} \eta$.

We will also consider the action of the complex Lie group $S O(n, \mathbb{C})$ on $\mathfrak{s o}(n, \mathbb{C}) \simeq \Lambda^{2} \mathbb{C}^{n}$.

Definition 2.9. Let $S$ be a subset of the complex Lie algebra $\mathfrak{s o}(n, \mathbb{C}) \simeq$ $\Lambda^{2} \mathbb{C}^{n}$ which is invariant under the action of $S O(n, \mathbb{C})$. The nonnegative curvature cone

$$
\mathcal{C}(S)=\left\{\mathrm{R} \in S_{B}^{2} \Lambda^{2} \mathbb{R}^{n} \mid\langle\mathrm{R}(\omega), \bar{\omega}\rangle \geq 0 \text { for all } \omega \in S\right\}
$$

is called the Wilking Cone associated with $S$.

Theorem 2.10 (Wil13). Any Wilking cone $\mathcal{C}(S)$ is Ricci flow invariant.

Sketch of proof: Let $\mathrm{R} \in \partial \mathcal{C}(S)$, and let $\omega$ be any element of $S$ such that $\langle\mathrm{R} \omega, \bar{\omega}\rangle$. We need to show that $\langle Q(\mathrm{R}) \omega, \bar{\omega}\rangle \geq 0$. We obviously have that $\left\langle\mathrm{R}^{2} \omega, \bar{\omega}\right\rangle=\langle\mathrm{R} \omega, \overline{\mathrm{R} \omega}\rangle \geq 0$.

We now show that $\left\langle\mathrm{R}^{\#} \omega, \bar{\omega}\right\rangle \geq 0$. Let $\eta$ be any element of $\mathfrak{s o}(n, \mathbb{C})$, and consider the function:

$$
t \mapsto\left\langle\operatorname{RAd}_{e^{t \eta}} \omega, \overline{\operatorname{Ad}_{e^{t \eta}} \omega}\right\rangle .
$$

It is nonnegative and attain its minimum at $t=0$. Differentiating twice and evaluating at $t=0$, we get that:

$$
\left\langle\mathrm{R}\left(\operatorname{ad}_{\eta} \operatorname{ad}_{\eta} \omega, \bar{\omega}\right\rangle+2\left\langle\operatorname{Rad}_{\eta} \omega, \operatorname{ad}_{\bar{\eta}} \bar{\omega}\right\rangle+\left\langle\mathrm{R} \omega, \operatorname{ad}_{\bar{\eta}} \operatorname{ad}_{\bar{\eta}} \bar{\omega}\right\rangle \geq 0 .\right.
$$

Replacing $\eta$ by $i \eta$ and summing we get that:

$$
\left\langle\operatorname{Rad}_{\eta} \omega, \operatorname{ad}_{\bar{\eta}} \bar{\omega}\right\rangle \geq 0 \text {. }
$$

Hence:

$$
\left\langle\operatorname{Rad}_{\omega} \eta, \operatorname{ad}_{\bar{\omega}} \bar{\eta}\right\rangle \geq 0
$$

This shows two things. First the Hermitian operator $-\operatorname{ad}_{\bar{\omega}} \circ \mathrm{R} \circ \operatorname{ad}_{\omega}$ and its conjugate $\mathrm{L}=-\operatorname{ad}_{\omega} \circ \mathrm{R} \circ \operatorname{ad}_{\bar{\omega}}$ are nonnegative. Second, $\mathrm{R}$ is nonnegative as an Hermitian operator on the image of $\operatorname{ad}_{\omega}$ (which contains the image of L).

These two things together imply that:

$$
\left\langle\mathrm{R}^{\#} \omega, \bar{\omega}\right\rangle=-\frac{1}{2} \operatorname{trace}(\mathrm{L} \mathrm{R})=-\frac{1}{2} \operatorname{trace}(\mathrm{R} \mathrm{L}) \geq 0 .
$$

Let us see now how this can be used to recover the Ricci flow invariance of various curvature cones (see Wil13 for more details):

- Choosing $S$ to be the whole $\mathfrak{s o}(n, \mathbb{C})$, we recover the invariance of $\mathcal{C}_{P C O}$. 
- If we let $S=\left\{\omega \in \mathfrak{s o}(n, \mathbb{C}) \mid \omega^{2}=0\right\}$, we get the invariance of $\mathcal{C}_{2 P C O}$.

- With $S=\left\{\omega \in \mathfrak{s o}(n, \mathbb{C}) \mid \omega^{2}=0, \operatorname{rank} \omega=2\right\}$, we have the invariance of $\mathcal{C}_{I C}$.

- Letting $S=\left\{\omega \in \mathfrak{s o}(n, \mathbb{C}) \mid \omega^{3}=0, \operatorname{rank} \omega=2\right\}$, we directly get the invariance of $\mathcal{C}_{I C 1}$.

- Finally, with $S=\{\omega \in \mathfrak{s o}(n, \mathbb{C}) \mid \operatorname{rank} \omega=2\}$, we obtain the invariance of $\mathcal{C}_{I C 2}$.

\subsection{One parameter families and differentiable sphere theo- rems}

Following the seminal work of Hamilton, one of the great successes of Ricci flow has been the proof of "differentiable sphere theorems" under various positive curvature assumptions. All these theorems are of the following form: "Let $\left(M, g_{0}\right)$ be a compact manifold with positive $\mathcal{C}$-curvature, then $M$ admits a constant sectional curvature metric and is thus diffeomorphic to a spherical space form".

The proof of this kind of theorem using Ricci flow has a mandatory step, the construction of a so called pinching set:

Definition 2.11. A closed convex $O(n)$-invariant $F \subset S_{B}^{2} \Lambda^{2} \mathbb{R}^{n}$ is a called a pinching set if:

- It is Ricci flow invariant.

- As $\lambda$ goes to $0, \lambda F$ converge $\}^{1}$ to $\mathbb{R}_{+} \mathrm{I}$.

Remark 2.12. A pinching set has to be a subset of the half space $\{\mathrm{R} \mid$ trace $\mathrm{R}>$ $0\}$.

Definition 2.13. A curvature cone $\mathcal{C}$ is said to have the pinching property if for any compact $K$ contained in the interior of $\mathcal{C}$, there is a pinching set $F$ containing $K$.

The interest of these definitions lies in the following proposition:

Proposition 2.14 ([Ham86, [BW08]). Let $\mathcal{C}$ be curvature cone with the pinching property, then any compact manifold with positive $\mathcal{C}$-curvature admits a metric with constant positive sectionnal curvature.

Sketch of proof: Let $\left(M, g_{0}\right)$ be a compact manifold with positive $\mathcal{C}$-curvature. By compactness of $M$, there exist a compact $K$ contained in the interior of $\mathcal{C}$ such that $R_{g_{0}}(x) \in K$ for all $x \in M$.

\footnotetext{
${ }^{1}$ By this we mean that for every compact $K \subset S_{B}^{2} \Lambda^{2} \mathbb{R}^{n},(\lambda F) \cap K$ converges in the Hausdorff topology to $\left(\mathbb{R}_{+}\right) \mathrm{I} \cap K$.
} 


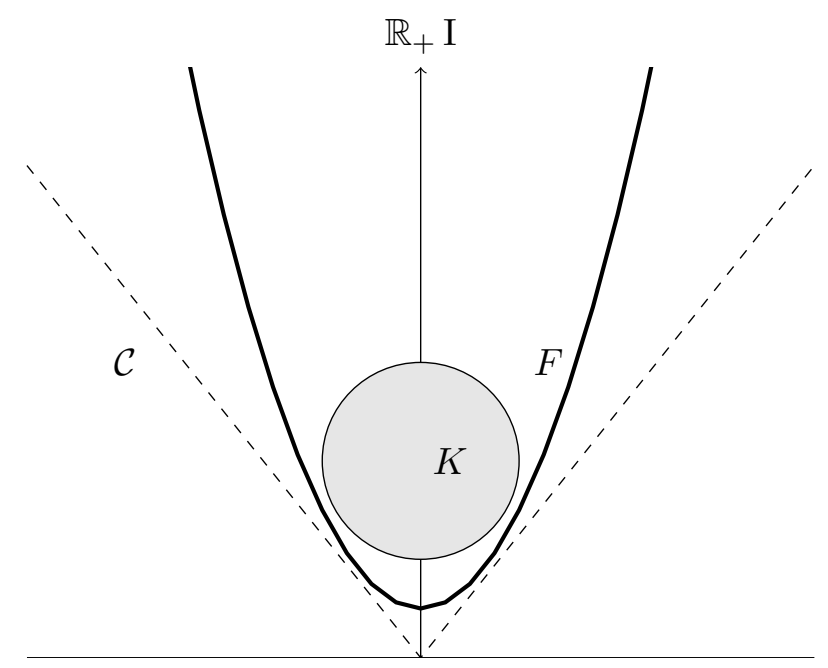

Figure 2: A pinching set $F$ containing a compact $K$ inside a cone $\mathcal{C}$ with the pinching property.

Since $g_{0}$ has positive scalar curvature, the Ricci flow $g(t)$ starting at $g_{0}$ is defined only on some interval $[0, T)$ where $T<\infty$ and the curvature of $g(t)$ blows up as $t \rightarrow T$. We consider a sequence of times $t_{i}<T$ converging to $T$, and points $x_{i} \in M$ where the maximum of the norm of the curvature tensor is attained. We let $C_{i}=\left|\mathrm{R}_{g\left(t_{i}\right)}\left(x_{i}\right)\right|$ and consider the sequence of parabolic blow ups centered at $x_{i}$ :

$$
g_{i}(t)=C_{i} g_{i}\left(t_{i}+\frac{t}{C_{i}}\right) .
$$

Perelman's no local collapsing theorem ensures that this sequence of Ricci flow converges, up to a subsequence, to some limiting Ricci flow $\left(M_{\infty}, g_{\infty}(t)\right)$ defined on some time interval containing 0 .

Let $F$ be a pinching set containing $K$, since $F$ is Ricci flow invariant, the curvature operator of of $g(t)$ satisfies $\mathrm{R}_{g(t)}(x) \in F$ for every $x \in M$ and $t \in[0, T)$, thus the curvature operator of $g_{i}(t)$ satisfies:

$$
\mathrm{R}_{g_{i}(t)}=\frac{1}{C_{i}} \mathrm{R}_{g\left(t_{i}+\frac{t}{K_{i}}\right)} \in \frac{1}{C_{i}} F .
$$

Letting $i$ go to infinity, we get that $\mathrm{R}_{g_{\infty}(t)} \in \mathbb{R}_{+}$I. Hence by Schur Lemma, $\left(M_{\infty}, g_{\infty}(t)\right)$ has constant positive sectional curvature, end is thus compact. The compactness of $M_{\infty}$ implies that $M$ is diffeomorphic to $M_{\infty}$.

Remark 2.15. One should note that this proposition alone doesn't show that the Ricci flow converges (after a suitable normalisation) to a metric of constant curvature, this can actually be achieved but requires more work see for instance the book [Bre10] by Brendle. 


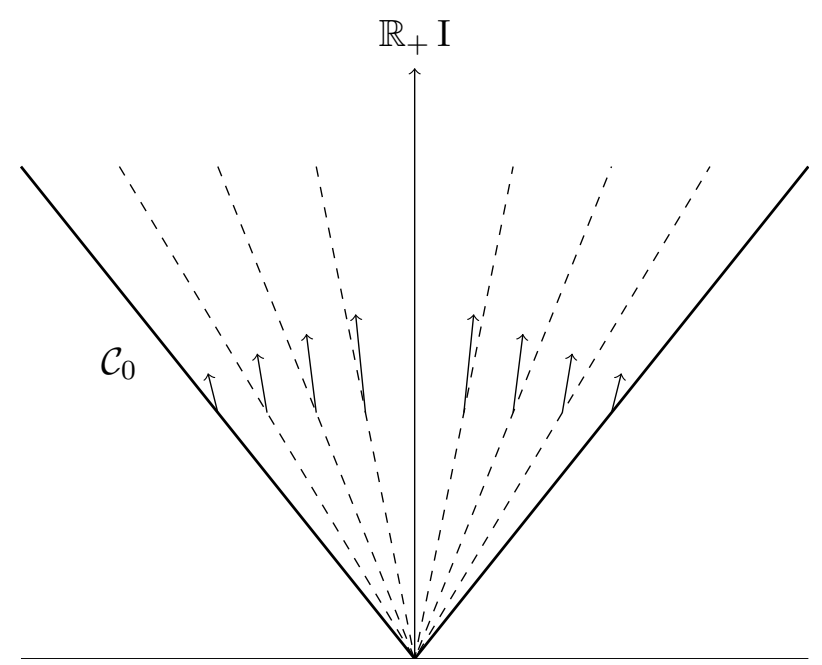

Figure 3: A pinching family

The problem is thus reduced to the construction of suitable pinching sets. A powerful method to achieve this is to build "pinching families" of curvature cones, which are 1-parameter families $\left(\mathcal{C}_{s}\right)_{s \in[0,1)}$ of curvature cones which start at the curvature condition under consideration and pinch towards the curvature cone $\mathbb{R}_{+} I$, which corresponds to constant positive sectional curvature.

Definition 2.16. A one parameter family $\left(\mathcal{C}_{s}\right)_{s \in[0,1)}$ of nonnegative curvature cones is called a pinching family if:

- $s \mapsto \mathcal{C}_{s}$ is continuous.

- as $s \rightarrow 1, \mathcal{C}_{s}$ converges to $\mathbb{R}_{+} \mathrm{I}$.

- for each $s>0$, every $\mathrm{R} \in \mathcal{C}_{s} \backslash\{0\}$ has positive trace.

- every $\mathcal{C}_{s}$ is Ricci flow invariant. Moreover for any $s>0$ and any non zero $\mathrm{R} \in \partial \mathcal{C}_{s}, Q(\mathrm{R})$ belongs to the interior of $T_{\mathrm{R}} \mathcal{C}_{s}$. 2

The idea can be tracked back to Hamilton's first paper on the Ricci flow of 3-manifolds [Ham82, although it was not really stressed. However, the definition we give comes from the work of Boehm and Wilking [BW08]. In this important paper, Boehm and Wilking also show the following proposition, which says that the existence of a pinching family is actually enough to ensure the existence of a pinching set:

Theorem 2.17. Let $\left(\mathcal{C}_{s}\right)_{s \in[0,1)}$ be a pinching family. Then $\mathcal{C}_{0}$ has the pinching property.

\footnotetext{
${ }^{2}$ One could say that $Q$ points strictly towards the inside of $\mathcal{C}_{s}$.
} 
Remark 2.18. From a PDE point of view, the strength of this result can be seen in the following way: it allows to build from a family of scale invariant estimate (the pinching family) a self improving non scale invariant estimate (the pinching set). Usually, non scale invariant estimates are harder to obtain.

Let us now give some examples of pinching families, the first example is specific to dimension 3, and is implicit in Hamilton's original work:

Proposition 2.19 ([am82]). The curvature cones

$$
\mathcal{C}_{s}=\left\{\mathrm{R} \in S_{B}^{2} \Lambda^{2} \mathbb{R}^{3} \mid \rho(\mathrm{R}) \geq \frac{2 s}{3}(\text { trace } \mathrm{R}) \text { id } \geq 0\right\}
$$

for $s \in[0,1)$ form a pinching family such that $\mathcal{C}_{0}=\{\mathrm{R} \mid \rho(\mathrm{R}) \geq 0\}$.

This gives a possible proof of Hamilton's result: any compact 3-manifold with positive Ricci curvature admits a metric with constant sectional curvature.

Most of the differentiable sphere theorems obtained through Ricci flow can be reduced to the construction of suitable pinching families. (See for instance [Hui85], Mar94, BW08], BS09.)

The older previously cited works ([Hui85], Mar94]) build their pinching families through ad-hoc constructions. A significant step forward was made by Boehm and Wilking in [BW08]: they used $O(n)$-equivariant transformations formations

$$
\ell_{a, b}(\mathrm{R})=\mathrm{R}_{\mathrm{I}}+a \mathrm{R}_{0}+b \mathrm{R}_{\mathcal{W}}
$$

to build pinching families from previously known Ricci flow invariant curvature cones.

More precisely, they considered, for a fixed Ricci flow invariant curvature cone $\hat{\mathcal{C}}$, the cones:

$$
\mathcal{C}_{s}=\left\{\mathrm{R} \mid \ell_{a(s), b(s)}(\mathrm{R}) \in \hat{\mathcal{C}} \text { and } \rho(\mathrm{R}) \geq c(s)(\text { trace } \mathrm{R}) \text { id } \geq 0\right\}
$$

and showed that, for suitable choices of $a(s), b(s)$ and $c(s)$, for $\hat{\mathcal{C}}=\mathcal{C}_{2 P C O}$, the cones $\mathcal{C}_{s}$ form a pinching family.

This construction has been re-used by Brendle and Schoen in their proof of the differentiable sphere theorem for $\frac{1}{4}$-pinched manifold to build a pinching family starting at the cone $\mathcal{C}_{I C 2}$, see BS09.

Let us end this section by mentioning Brendle's sphere theorem, in the language we have developed it can phrased as: the nonnegative curvature cone $\mathcal{C}_{I C 1}$ has the pinching property. However Brendle's proof is different from the strategy of proof we have outlined here: it doesn't show the existence of a pinching family starting at $\mathcal{C}_{I C 1}$, but directly show the existence of a pinching set. See [BS09] or [Bre10] for details. 


\section{Restrictions on Ricci flow invariant curvature cones}

The previous sections have stressed the importance of Ricci invariant curvature cones, and showed which kind of examples are available. Also, it should be noted that it is often computationally hard to prove that a curvature cone is invariant.

It is thus important to find necessary conditions satisfied by every Ricci flow invariant curvature cones, so that as many as possible curvature cones one can think of can be discarded in advance without any computation.

This idea has not been investigated until recently, and only a handful of results are available now. We present them here.

\subsection{Dimension independent restrictions}

The first restrictions that were obtained dealt only with Wilking cones. These can be found in GMS13 by Gururaja, Maity and Seshadri.

Theorem 3.1. Let $\mathcal{C} \subset S_{B}^{2} \Lambda^{2} \mathbb{R}^{n}$ be a Wilking with $n \geq 5$, then $\mathcal{C}$ is contained in the cone $\mathcal{C}_{I C}$ of manifolds with nonnegative isotropic curvature.

The proof takes advantage the Wilking cone property by using some advanced results on the orbits of the adjoint action of Lie groups. In the same paper, the Wilking cones which had the pinching property were also characterized:

Theorem 3.2. Let $\mathcal{C} \subset S_{B}^{2} \Lambda^{2} \mathbb{R}^{n}$ be a Wilking with $n \geq 5$, assume that $\mathcal{C}$ has the pinching property, then $\mathcal{C}$ is contained in the cone $\mathcal{C}_{I C 1}$.

The next results do not require the Wilking assumptions. They come from the article RS13a, by H. Seshadri and the author.

Theorem 3.3. Let $\mathcal{C} \subset S_{B}^{2} \Lambda^{2} \mathbb{R}^{n}, n \geq 4$, be a Ricci flow invariant curvature cone, assume $\mathcal{C}$ contains $\mathcal{W}$, the space of Ricci flat curvature operators, then $\mathcal{C}$ is either the cone $\mathcal{C}_{\text {Scal }}$ or the whole space $S_{B}^{2} \Lambda^{2} \mathbb{R}^{n}$.

Remark 3.4. For representation theoretic reasons, any curvature cone which contains a non-vanishing tensor $W \in \mathcal{W}$ automatically contains the whole space $\mathcal{W}$. This will be useful in the applications.

Remark 3.5. Recall that nonnegative Ricci curvature is not preserved under Ricci flow in dimension 4 and above (see Section 2.1). It is thus quite natural to ask wether some weaker condition which is implied by nonnegative Ricci curvature is preserved, as the results proven using this condition would apply to the important class of manifolds with nonnegative Ricci curvature. The above result shows the only condition one can get following this idea is the "nonnegative scalar curvature" condition. 


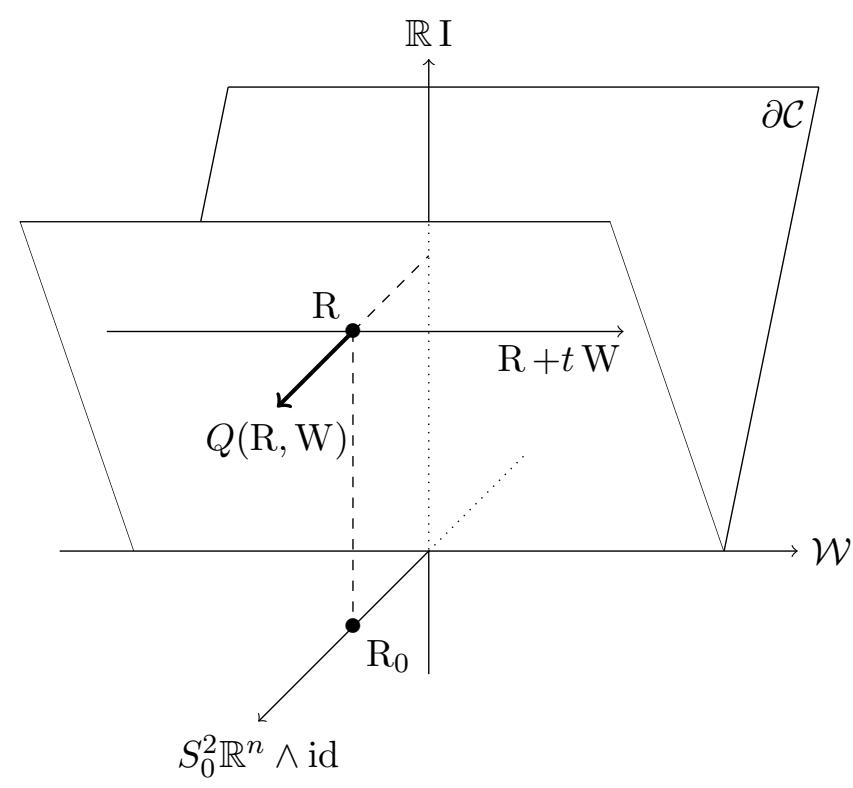

Figure 4: A cone which contains $\mathcal{W}$ must contain $S_{0}^{2} \mathbb{R}^{n} \wedge$ id.

Sketch of proof: We argue by contradiction. Let $\mathcal{C}$ be a Ricci flow invariant curvature cone satisfying the hypothesis of the theorem but isn't one of the cones $\mathcal{C}_{\text {Scal }}$ or $S_{B}^{2} \Lambda^{2} \mathbb{R}^{n}$. Examining the decomposition of $S_{B}^{2} \Lambda^{2} \mathbb{R}^{n}$ as representation of $O(n)$ into irreducible components, we see that these hypothesis imply that $\mathcal{C}$ doesn't contain any non zero tensor in $S_{0}^{2} \mathbb{R}^{n}$ (see Figure 4).

The proof is based on the following observation: let $\mathrm{R}$ be a curvature operator in $\partial \mathcal{C}$, and $\mathrm{W}$ be a curvature operator in $\mathcal{W}$, then $\mathrm{R}+t \mathrm{~W} \in \partial \mathcal{C}$ and the Ricci flow invariance gives that:

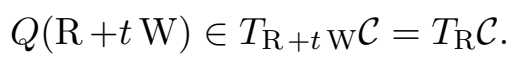

We now compute:

$$
Q(\mathrm{R}+t \mathrm{~W})=Q(\mathrm{R})+2 t Q(\mathrm{R}, \mathrm{W})+t^{2} Q(\mathrm{~W}) \in T_{\mathrm{R}} \mathcal{C} .
$$

We remark that $Q(\mathrm{~W}) \in \mathcal{W} \subset T_{\mathrm{R}} \mathcal{C}$. Since $\mathcal{W}$ is a vector space, $-Q(\mathrm{~W}) \in$ $T_{\mathrm{R}} \mathcal{C}$. We conclude that $Q(\mathrm{R})+2 t Q(\mathrm{R}, \mathrm{W})$ belongs to $T_{\mathrm{R}} \mathcal{C}$ as a conical combination of two elements of $T_{\mathrm{R}} \mathcal{C}$. Dividing by $2 t$ and letting $t$ go to infinity, we get that $Q(\mathrm{R}, \mathrm{W}) \in T_{\mathrm{R}} \mathcal{C}$.

To end the proof we produce explicit operators $\mathrm{R}_{0} \in S_{0}^{2} \mathbb{R}^{n} \wedge \mathrm{id}, \mathrm{W} \in \mathcal{W}$ such that:

- There exists $a>0$ such that $\mathrm{R}=\mathrm{I}+a \mathrm{R}_{0}$ belongs to $\mathcal{C}$ but $\mathrm{R}=$ $\mathrm{I}+(a+\varepsilon) \mathrm{R}_{0}$ is outside of $\mathcal{C}$ for every $\varepsilon>0$.

- $Q(\mathrm{R}, \mathrm{W})=\mathrm{R}_{0}$. 
This implies that $Q(\mathrm{R}, \mathrm{W})$ which should be in $T_{\mathrm{R}} \mathcal{C}$, points outside $\mathcal{C}$, this contradicts the Ricci flow invariance of $\mathcal{C}$. See Figure 4 ,

The following corollary can also be found in [RS13a]:

Corollary 3.6. Let $\mathcal{C} \subset S_{B}^{2} \Lambda^{2} \mathbb{R}^{n}, n \geq 4$, be a Ricci flow invariant curvature cone, assume $\mathcal{C}$ contains in its interior the curvature operator of a compact Einstein symmetric space with non constant nonnegative sectionnal curvature (such as $\mathbb{C P}^{n}$ or $\mathbb{S}^{n} \times \mathbb{S}^{n}$ ), then $\mathcal{C}$ is either the cone $\mathcal{C}_{\text {Scal }}$ or the whole space $S_{B}^{2} \Lambda^{2} \mathbb{R}^{n}$.

Remark 3.7. This theorem explains the following observation, whereas the complex projective space $\mathbb{C P}^{n}$ is the second example one usually gives when asked for a manifolds of positive curvature, when one considers Ricci flow invariant curvature conditions, it only has nonnegative curvature at best. The above theorem shows that "nonnegative scalar curvature" is the only Ricci flow invariant curvature condition for which $\mathbb{C P}^{n}$ is positively curved.

Remark 3.8. As in every even dimension $2 n$, with $n \geq 2, \mathbb{C P}^{n}$ is an Einstein symmetric space with non constant positive sectional curvature, the above theorem shows that in even dimensions there is no Ricci flow invariant curvature cone which contains the cone of operators with nonnegative sectional curvature.

Sketch of proof: Let $\mathrm{R}$ be the curvature operator of an Einstein symmetric space, a simple computation shows that $Q(\mathrm{R})=\lambda \mathrm{R}$ (CITE BRENDLE). Since $\mathrm{R}$ is Einstein, we can decompose it as $\mathrm{R}=\mathrm{R}_{\mathrm{I}}+\mathrm{R}_{\mathcal{W}}$. One then observes that solutions to $\mathrm{R}^{\prime}=Q(\mathrm{R})$ whose initial condition is of the form $\alpha \mathrm{R}_{\mathrm{I}}+\beta \mathrm{R}_{\mathcal{W}}$ remain of this form. The components $\alpha$ and $\beta$ evolve according to the differential equations:

$$
\left\{\begin{array}{l}
\alpha^{\prime}=\lambda \alpha^{2} \\
\beta^{\prime}=\lambda \beta^{2} .
\end{array}\right.
$$

This system can be explicitly integrated and one sees from this that that all solutions escape to infinity and fall into three classes (see Figure 5):

- The diagonal is the trajectory of a solution.

- When the initial condition lies below the diagonal, the trajectory is part of an hyperbola which is asymptotic to a horizontal line.

- When the initial condition lies above the diagonal, the trajectory is part of an hyperbola which is asymptotic to a vertical line.

Now if we assume that $\mathrm{R}$ is in the interior of $\mathcal{C}$, then $\mathcal{C}$ must contain some trajectory whose initial condition is below the diagonal, since this trajectory will be asymptotic to an horizontal line, the fact that $\mathcal{C}$ is closed and convex 
implies that $\mathcal{C}$ must contain the " $x$-axis". Hence $\mathcal{C}$ contains $\mathrm{R}_{\mathcal{W}}$ which is not zero because we assumed that $\mathrm{R}$ is the curvature operator of an Einstein symmetric space with non constant sectional curvature. Thus $\mathcal{C}$ contains $\mathcal{W}$ and we can apply the previous theorem to conclude that $\mathcal{C}$ is one of the cones $\mathcal{C}_{\text {Scal }}$ or the whole space $S_{B}^{2} \Lambda^{2} \mathbb{R}^{n}$.

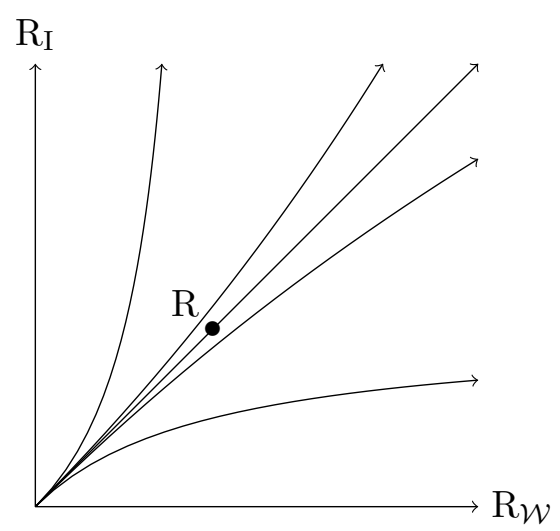

Figure 5: Trajectories of $\mathrm{R}^{\prime}=Q(\mathrm{R})$ in the $\left(\mathrm{R}_{\mathrm{I}}, \mathrm{R}_{\mathcal{W}}\right)$ plane, where $\mathrm{R}$ is the curvature operator of an Einstein symmetric space with non constant sectional curvature. (Equation (44).)

\subsection{The case of dimension 4}

Besides these dimension independent restrictions, we can prove much stronger results in dimension 4.

When we consider $S_{B}^{2} \Lambda^{2} \mathbb{R}^{4}$ as a representation of $O(4, \mathbb{R})$ we have the usual decomposition of $S_{B}^{2} \Lambda^{2} \mathbb{R}^{4}$ into irreducible representations:

$$
S_{B}^{2} \Lambda^{2} \mathbb{R}^{4}=\mathbb{R} \mathrm{I} \oplus\left(S_{0}^{2} \mathbb{R}^{4} \wedge \mathrm{id}\right) \oplus \mathcal{W} .
$$

However, when one considers only the action of $S O(4, \mathbb{R})$ on $S_{B}^{2} \Lambda^{2} \mathbb{R}^{4}$, the component $\mathcal{W}$ is no longer irreducible and splits as $\mathcal{W}=\mathcal{W}_{+} \oplus \mathcal{W}_{-}$, the self dual and anti self dual Weyl tensors. This fact is specific to dimension 4 and can be linked to the fact that the Lie algebra $\mathfrak{s o}(n, \mathbb{R})$ is irreducible except when $n=4$ where we have the splitting: $\mathfrak{s o}(4, \mathbb{R}) \simeq \mathfrak{s o}(3, \mathbb{R}) \oplus \mathfrak{s o}(3, \mathbb{R})$.

This reducibility makes it interesting to weaken the requirements made for curvature cones:

Definition 3.9. An oriented curvature cone is a closed convex cone $\mathcal{C} \subset$ $S_{B}^{2} \Lambda^{2} \mathbb{R}^{4}$ which is invariant under the action of $S O(4, \mathbb{R})$.

Remark 3.10. We define a nonnegative oriented curvature cone and Ricci flow invariant oriented curvature cone in the same way as their unoriented counterparts. The main difference we will encounter is that $\mathcal{C}$-nonnegative curvature will now only make sense for oriented Riemannian 4-manifolds. 
Let us give an example of a nonnegative oriented curvature cone which is not a curvature cone:

Definition 3.11. A curvature operator $\mathrm{R} \in S_{B}^{2} \Lambda^{2} \mathbb{R}^{4}$ is to be positively half- $\overline{P I C}$ (in short $\overline{P I C}_{+}$) if and only if for every positively oriented orthonormal 4-frame $\left(e_{1}, e_{2}, e_{3}, e_{4}\right)$ :

$$
\mathrm{R}_{1313}+\mathrm{R}_{1414}+\mathrm{R}_{2323}+\mathrm{R}_{2424}-2 \mathrm{R}_{1234} \geq 0 .
$$

The oriented curvature cone of all such curvature operators will be denoted by $\mathcal{C}_{I C_{+}}$.

Similarly, the cone $\mathcal{C}_{I C_{-}}$of negatively half- $\overline{P I C}$ curvature operators is defined by requiring inequality (5) to hold for every negatively oriented 4frame.

Remark 3.12. The cones $\mathcal{C}_{I C_{+}}$and $\mathcal{C}_{I C_{-}}$lie between the cones $\mathcal{C}_{\text {Scal }}$ and $\mathcal{C}_{I C}$, and are Wilking cones. It can also be proven that they are Ricci flow invariant.

Example 3.13. Besides PIC 4-manifolds such as $\mathbb{S}^{4}$ and $\mathbb{S}^{3} \times \mathbb{R}$, another example of 4 -manifold with strictly $\mathcal{C}_{I C_{+}}$-positive curvature is given by $\overline{\mathbb{C P}}^{2}$, the complex projective plane with the Fubini-Study metric and reversed orientation.

In [RS13b], H. Seshadri and the author used the results from [RS13a] to prove that the cones $\mathcal{C}_{I C_{+}}$and $\mathcal{C}_{I C_{-}}$enjoy some kind of maximality among oriented Ricci flow invariant nonnegative curvature cones in dimension 4.

Theorem 3.14. Let $\mathcal{C} \subset S_{B}^{2} \Lambda^{2} \mathbb{R}^{4}$ be an oriented curvature cone.

- If $\mathcal{C} \subsetneq \mathcal{C}_{\text {Scal }}$, then $\mathcal{C}$ is contained in either $\mathcal{C}_{I C_{+}}$or $\mathcal{C}_{I C_{-}}$.

- If $\mathcal{C}$ is an (unoriented) curvature cone and $\mathcal{C} \subsetneq \mathcal{C}_{\text {Scal }}$, then $\mathcal{C}$ is contained in $\mathcal{C}_{I C}$.

\section{Open questions}

We end this survey by some open questions about Ricci flow invariant curvature cones.

We have proved in section 3.1 that, in dimension 4 and above, no Ricci flow invariant cone can contain every curvature operator with nonnegative Ricci curvature, except the cone $\mathcal{C}_{\text {Scal }}$. We also saw that, in even dimension greater than 4, no Ricci flow invariant cone can contain every curvature operator with nonnegative sectional curvature, except the cone $\mathcal{C}_{\text {Scal }}$. It is thus natural to ask:

Question 4.1. In odd dimension 5 and above, does there exist Ricci flow invariant cones which contain every curvature operator with nonnegative sectional curvature? 
In a similar but slightly more ambitious trend, one might want to find the biggest non trivial Ricci flow invariant cone. We already have a candidate for this:

Question 4.2. What are the biggest Ricci flow invariant curvature cones? Is there any curvature cone, other that $\mathcal{C}_{\text {Scal }}$ which is not contained the cone $\mathcal{C}_{I C}$ ?

The last section answers this question affirmatively in dimension 4 (if one ignores oriented curvature cones). We also saw in the beginning of section 3.1 that the answer is yes when the investigation is restricted to Wilking cones.

Answering the following questions would make the matter of finding sphere theorems using Ricci flow closed:

Question 4.3. Does there exist a Ricci flow invariant curvature cone with the pinching property which is not contained in $\mathcal{C}_{I C 1}$ ? Is there a maximal Ricci flow invariant curvature cone with the pinching property?

We have seen in section 3.1 that $\mathcal{C}_{I C 1}$ is maximal among Wilking cones with the pinching property.

A Ricci flow with surgeries has been constructed for general closed 3manifolds by Perelman and for closed PIC 4-manifolds by Hamilton. It is still unknown wether or not a Ricci flow with surgeries can be built for PIC manifolds of arbitrary dimensions. Next question is a possible step towards this goal:

Question 4.4. Can one pinch the cone $\mathcal{C}_{I C}$ towards a smaller cone? For instance, can one find a continuous family of cones $\left(\mathcal{C}_{s}\right)_{s \in[0,1]}$ such that:

- $\mathcal{C}_{0}=\mathcal{C}_{I C}$,

- for each $s \in(0,1), Q$ points strictly inwards $\mathcal{C}_{s}$,

- $\mathcal{C}_{1}$ is a "geometrically constraining" cone, such as $\mathcal{C}_{P C O}$ or $\mathcal{C}_{I C 1}$.

The existence of such a "generalized pinching family" should imply that blow up of singularities of PIC Ricci flows should have $\mathcal{C}_{1}$-nonnegative curvature, which would make their study easier. This would play a role similar to the role of the Hamilton-Ivey estimate in dimension 3.

It would also be interesting to see how these results can be adapted to the Kaehler-Ricci flow. Note first that Wilking's construction -works in the Kaehler case with only minor modifications (this is treated in [Wil13]). However, for results in the spirit of section 3 , it seems that a better understanding of how $Q$ interacts with the representation theory of Kaehler curvature operators. 


\section{References}

[AH11] Ben Andrews and Christopher Hopper. The Ricci flow in Riemannian geometry. A complete proof of the differentiable 1/4pinching sphere theorem. Berlin: Springer, 2011.

$\left[\mathrm{BBM}^{+} 10\right]$ Laurent Bessières, Gérard Besson, Sylvain Maillot, Michel Boileau, and Joan Porti. Geometrisation of 3-manifolds. Zürich: European Mathematical Society (EMS), 2010.

[Bre10] Simon Brendle. Ricci flow and the sphere theorem. Providence, RI: American Mathematical Society (AMS), 2010.

[BS09] Simon Brendle and Richard Schoen. Manifolds with 1/4-pinched curvature are space forms. J. Am. Math. Soc., 22(1):287-307, 2009 .

[BW08] Christoph Böhm and Burkhard Wilking. Manifolds with positive curvature operators are space forms. Ann. Math. (2), 167(3):1079-1097, 2008.

[Che91] Haiwen Chen. Pointwise 1/4-pinched 4-manifolds. Ann. Global Anal. Geom., 9(2):161-176, 1991.

[CK04] Bennett Chow and Dan Knopf. The Ricci flow: an introduction. Providence, RI: American Mathematical Society (AMS), 2004.

[CLN06] Bennett Chow, Peng Lu, and Lei Ni. Hamilton's Ricci flow. Providence, RI: American Mathematical Society (AMS), 2006.

[GMS13] H.A. Gururaja, Soma Maity, and Harish Seshadri. On Wilking's criterion for the Ricci flow. Math. Z., 274(1-2):471-481, 2013.

[Gro91] M. Gromov. Sign and geometric meaning of curvature. Rend. Semin. Mat. Fis. Milano, 61:9-123, 1991.

[Ham82] Richard S. Hamilton. Three-manifolds with positive Ricci curvature. J. Differ. Geom., 17:255-306, 1982.

[Ham86] Richard S. Hamilton. Four-manifolds with positive curvature operator. J. Differ. Geom., 24:153-179, 1986.

[Hui85] Gerhard Huisken. Ricci deformation of the metric on a Riemannian manifold. J. Differ. Geom., 21:47-62, 1985.

[KL08] Bruce Kleiner and John Lott. Notes on Perelman's papers. Geom. Topol., 12(5):2587-2855, 2008. 
[Mar94] Christophe Margerin. Une caractérisation optimale de la structure différentielle standard de la sphère en terme de courbure pour (presque) toutes les dimensions. I: Les énoncés. C. R. Acad. Sci., Paris, Sér. I, 319(6):605-607, 1994.

[Max11] Davi Maximo. Non-negative Ricci curvature on closed manifolds under Ricci flow. Proc. Am. Math. Soc., 139(2):675-685, 2011.

[MM88] Mario J. Micallef and John Douglas Moore. Minimal two-spheres and the topology of manifolds with positive curvature on totally isotropic two-planes. Ann. Math. (2), 127(1):199-227, 1988.

[MT07] John Morgan and Gang Tian. Ricci flow and the Poincaré conjecture. Providence, RI: American Mathematical Society (AMS); Cambridge, MA: Clay Mathematics Institute, 2007.

[Ngu10] Huy T. Nguyen. Isotropic curvature and the Ricci flow. Int. Math. Res. Not., 2010(3):536-558, 2010.

[Per02] G. Perelman. The entropy formula for the Ricci flow and its geometric applications. ArXiv Mathematics e-prints, November 2002 .

[Per03a] G. Perelman. Finite extinction time for the solutions to the Ricci flow on certain three-manifolds. ArXiv Mathematics e-prints, July 2003.

[Per03b] G. Perelman. Ricci flow with surgery on three-manifolds. ArXiv Mathematics e-prints, March 2003.

[RS13a] T. Richard and H. Seshadri. Noncoercive Ricci flow invariant curvature cones. ArXiv e-prints, to appear in Proc. of American Mathematical Society, August 2013.

[RS13b] T. Richard and H. Seshadri. Positive isotropic curvature and self-duality in dimension 4. ArXiv e-prints, November 2013.

[Top06] Peter Topping. Lectures on the Ricci flow. Cambridge: Cambridge University Press, 2006.

[Wil13] Burkhard Wilking. A Lie algebraic approach to Ricci flow invariant curvature conditions and Harnack inequalities. J. Reine Angew. Math., 679:223-247, 2013. 